\title{
Relationship between Discordance of Low-Density Lipoprotein and Non-High-Density Lipoprotein Cholesterol and Risk Stratification in Acute Myocardial Infarction
}

\author{
Murat Eren, ${ }^{\circledR}$ Ozge Kurmus, ${ }^{\circledR}$ Turgay Aslan, ${ }^{1 \oplus}$ Kursat Akbuga, $^{1 \oplus}$ Hatice Tolunay ${ }^{2}{ }^{\circledR}$ \\ Ufuk University, ${ }^{1}$ Ankara - Turkey \\ Gulhane Education and Research Hospital, ${ }^{2}$ Ankara - Turkey
}

\section{Abstract}

Background: Sizeable proportion of patients have discordant low-density lipoprotein cholesterol (LDL-C) and nonhigh density lipoprotein cholesterol (NHDL-C). It has been shown that discordance of LDL-C and NHDL-C either underestimates or overestimates coronary risk.

Objectıve: We assessed whether this discordance has an impact on GRACE and TIMI risk scores in patients with acute myocardial infarction (AMI).

Methods: We retrospectively evaluated the data of 198 consecutive patients with AMI. Fasting serum lipid profiles were recorded, GRACE and TIMI scores were calculated. Patients were divided into 3 groups according to LDL-C and NHDL-C percentiles: Discordant group: LDL-C $<$ NHDL-C ( $n=38)$, concordant group: LDL-C=NHDL-C ( $\mathrm{n}=112)$ and discordant group LDL-C $>$ NHDL-C $(\mathrm{n}=48)$. GRACE and TIMI scores, mortality and cardiovascular events (heart failure, non-fatal myocardial infarction and angina) at sixth month were compared between these three groups. Differences between these groups were analyzed with One-way ANOVA or Kruskal-Wallis rank test, and with chi-square for percentages. Also, post hoc LSD or Conover-Iman's non-parametric multiple comparison test were used. A p value $<0.05$ was accepted as statistically significant.

Results: TIMI risk score didn't differ between discordant or concordant groups. Mean GRACE (death) and GRACE (death and MI) scores were higher in group with LDL-C $<$ NHDL-C than with LDL-C $=$ NHDL-C and LDL-C $>$ NHDL-C $(\mathrm{p}=0.029$ and 0.008 , respectively). Cardiovascular events and mortality at sixth month were not different among groups ( $\mathrm{p}=0.473$ and $\mathrm{p}=0.176$, respectively).

Conclusion: GRACE score was higher in discordant group with LDL-C $<$ NHDL-C, but there is no difference regarding TIMI scores between discordant and concordant groups in AMI patients.

Keywords: Atherosclerosis; Low Density Lipoprotein Receptor -Related Protein; Cholesteroç-HDL; Triglycerides; Cardiovascular Diseases/complications.

Introduction

Low-density lipoprotein-cholesterol (LDL-C) is the primary target of lipid lowering therapy to prevent atherosclerotic events. ${ }^{1}$ However, despite the LDLlowering therapy, some acute myocardial infarction (AMI) patients continue to have residual risk. ${ }^{2}$

Non-high-density lipoprotein-cholesterol (NHDL-C) is recommended as a secondary target for lipid management. ${ }^{1}$ There is a growing body of evidence that NHDL-C is a better predictor of major cardiovascular events after AMI. ${ }^{3}$ However, not all patients have concordant LDL-C and NHDL-C levels. It has been shown that discordance of LDL-C and NHDL-C either underestimates or overestimates coronary risk. ${ }^{4}$

The GRACE and TIMI risk scores are widely used models to assess patients prognosis after acute coronary 
syndromes. ${ }^{5,6}$ They incorporate patient's characteristics, clinical and laboratory findings for risk stratification. In this study, we aimed to evaluate the relationship between discordance of LDL-C and NHDL-C and risk scores to find out whether the discordance of lipid parameters have impact on mortality after AMI.

\section{Methods}

This study assessed the data of 209 consecutive patients admitted to cardiology department with a diagnosis of acute myocardial infarction between January 2009 and June 2009. Among these, 11 patients were excluded because of incomplete data. Finally, we evaluated the data of 198 patients in our analysis. Exclusion criteria were as follows: age above 85 years old, clinical active infection, active malignancy, hematological or autoimmune disorders, receiving chemotherapy or steroids, cardiogenic shock, decompensated heart failure, arrhythmia with hemodynamic instability, and percutaneous coronary intervention or surgery within 30 days before admission. The assessed clinical parameters were age, gender and coronary risk factors. Hypertension was defined as systolic blood pressure $\geq 140 \mathrm{mmHg}$ and/ or diastolic blood pressure $\geq 90 \mathrm{mmHg}$ and/or current medication with antihypertensive drugs. Patients were defined as diabetic if they had been informed of this diagnosis before and had been using oral antidiabetic drugs or insulin treatment on admission. Body mass index was calculated as body weight in kilograms divided by the squared value of body height in meters $\left(\mathrm{kg} / \mathrm{m}^{2}\right)$.

Type of acute coronary syndrome, admission blood pressure and heart rate, ST- segment changes in ECG, Killip classification or presence of cardiac arrest at admission, and ejection fraction were evaluated. GRACE and TIMI scores were calculated from the clinical history, electrocardiogram, and laboratory values collected on admission. TIMI risk score represents the sum of seven variables including age, $>65$ years, $>3$ risk factors for coronary artery disease, use of aspirin in the past 7 days, known coronary artery stenosis $>50 \%, \geq 2$ episodes of angina within 24 hours, ST-segment deviation and elevated cardiac markers. One point is given to each of the variables present at the time of assessment. Higher risk score is correlated with increased incidence of death, myocardial infarction and revascularization. Grace score was calculated using GRACE 2.0 calculator at the time of admission by using the following variables: age, heart rate, systolic blood pressure, creatinine, cardiac arrest at admission, ST-segment deviation, elevated cardiac markers and Killip class. GRACE (death) represents in-hospital and 6-month mortality after a myocardial infarction and GRACE (death and MI) represents death or myocardial infarction from admission to six months after discharge. Also, in-hospital mortality and cardiovascular events at sixth month including angina, non-fatal myocardial infarction and heart failure were recorded. Data were obtained from patient files and hospital computer registry system. Also, 6-month mortality was assessed by contacting patients and their family with a telephone call.

The study was approved by the local Ethics Committee. Data from subjects were analyzed retrospectively.

\section{Laboratory Measurements}

Lipid measurements were performed on fasting blood samples that were taken before the angiography. Plasma concentrations of total cholesterol, LDL-C, HDL-C and triglyceride (TG) were measured with biochemistry analyzer (Abbott Architect c8000). The enzymatic colorimetric method was used for the quantitative determination of total cholesterol. Endpoint colorimetric method was used for the quantitative determination of HDL-C. Glycerol phosphate oxidase method was used for the quantitative determination of triglyceride. The Friedewald equation was used to calculate LDL-C.? NHDL-C was calculated as total cholesterol minus HDL-C.

\section{Statistical Analysis}

Continuous variables were expressed as mean \pm standard deviation (SD) for normal distributions and median (interquartile range) for skewed distributions. Categorical variables were defined as numbers and percentages. Whether the distribution of continuous variables was normal or not was determined by Kolmogorov-Smirnov test. Levene test was used for the evaluation of homogeneity of variances. Correlation between continuous variables including LDL-C and NHDL-C was examined with Spearman correlation analysis. Percentile distributions of LDL-C and NHDL-C were calculated. Discordance was determined based on the difference in the percentile of NHDL-C and percentile of LDL-C. Six percentile points was used as a cutoff so that the numbers of patients that would be classified as concordant or discordant were as close as possible. Patients were divided into 3 groups according to percentiles: Discordant group: LDL-C $<$ NHDL-C 
$(\mathrm{n}=38)$, concordant group: LDL-C=NHDL-C ( $\mathrm{n}=112)$ and discordant group LDL-C $>$ NHDL-C $(n=48)$. Differences between baseline characteristics of patients across these groups were analyzed with One-way ANOVA for normally distributed variables, with Kruskal-Wallis rank test for not normally distributed variables, and with chi-square for percentages. When the $\mathrm{p}$-value from One-Way ANOVA or Kruskal Wallis test statistics were statistically significant, post hoc LSD or Conover-Iman's non-parametric multiple comparison test were used to know which group differ from which others. A $p$ value $<0.05$ was accepted as statistically significant. Data analyses were performed by using SPSS for Windows, version 22.0 (SPSS Inc., Chicago, IL, United States).

\section{Results}

The mean age of study population was $62.7 \pm 10.7$ years and of the 198 patients $73.7 \%$ were males. More than half of the patients had hypertension, $30 \%$ had diabetes mellitus, $61 \%$ had any smoking history and $38 \%$ had dyslipidemia. Baseline characteristics are presented in Table 1. Fifty-one and a half percent of the patients had NSTEMI and $48.5 \%$ had STEMI. Mean difference between NHDL-C and LDL was $31.9 \pm 20.5 \mathrm{mg} / \mathrm{dl}$. LDL-C levels were strongly and positively correlated with NHDL-C levels $(\mathrm{r}=0.858$, $\mathrm{p}<0.001$ ). In-hospital mortality rate was $2 \%$. In 10 patients, death occurred during six months follow-up.

To further evaluate the characteristics of patients with discordance and concordance of LDL-C and NHDL-C, we classified patients into 3 subgroups according to percentiles: Discordant group: LDL-C $<$ NHDL-C $(\mathrm{n}=38)$, concordant group: LDL-C=NHDL-C $(\mathrm{n}=112)$ and discordant group LDL-C $>$ NHDL-C $(n=48)$ (Figure 1). Gender, age, smoking history, percentage of patients with hypertension or percentage of patients with dyslipidemia were not different among the groups. Patients in the discordant group LDL-C $<$ NHDL-C, had the highest prevalence of diabetes mellitus ( $\mathrm{p}=0.013$ ). Median TIMI score was not different among groups. Mean GRACE (death) score and GRACE (death and MI) score were highest in the discordant group LDL-C $<$ NHDL-C than in the concordant group LDL-C=NHDL-C and the discordant group LDL$\mathrm{C}>$ NHDL-C ( $\mathrm{p}=0.029$ and $\mathrm{p}=0.008$, respectively) (Table 2). Cardiovascular events and mortality at sixth month were not different among groups $(\mathrm{p}=0.473$ and $\mathrm{p}=0.176$, respectively). Because of the small number of in-hospital deaths in each group, analysis of these subgroups regarding in-hospital mortality was not possible.

\section{Discussion}

In the present study, we assessed the cross-sectional association between LDL-C and NHDL-C discordance and Grace and TIMI scores. Median TIMI score was not different between discordant and concordant groups. Mean GRACE score was highest in the discordant group with LDL- $\mathrm{C}<\mathrm{NHDL}-\mathrm{C}$.

Table 1 - Baseline characteristics of study population

\begin{tabular}{|c|c|}
\hline Characteristics & \\
\hline Clinical characteristics & \\
\hline Gender (\% male) & 73.7 \\
\hline $\begin{array}{l}\text { Age (years) } \\
\text { (mean } \pm \text { standart deviation) }\end{array}$ & $62.7 \pm 10.7$ \\
\hline Smoking (\%) & 61.6 \\
\hline Hypertension (\%) & 60.6 \\
\hline Diabetes (\%) & 30.3 \\
\hline Dyslipidemia (\%) & 37.9 \\
\hline
\end{tabular}

Body mass index $\left(\mathrm{kg} / \mathrm{m}^{2}\right)$

(mean \pm standart deviation)

$26.8 \pm 4.1$

Biochemical analysis

(mean \pm standart deviation)

Total cholesterol (mg/dl)

$185.8 \pm 41.4$

$\begin{array}{ll}\text { LDL-C }(\mathrm{mg} / \mathrm{dl}) & 116.7 \pm 33.3\end{array}$

$\begin{array}{ll}\mathrm{HDL}-\mathrm{C}(\mathrm{mg} / \mathrm{dl}) & 37.1 \pm 9.8\end{array}$

$\begin{array}{ll}\text { Triglyceride }(\mathrm{mg} / \mathrm{dl}) & 162.7 \pm 112.9\end{array}$

NHDL-C (mg/dl) $\quad 148.6 \pm 40.1$

Fasting glucose (mg/dl)

glucose $(\mathrm{mg} / \mathrm{dl})$

$116.6 \pm 42.1$

Creatinine $(\mathrm{mg} / \mathrm{dl})$

$1.1 \pm 0.55$

Risk scores

(mean \pm standart deviation)

Mean GRACE (death) score

$110.2+29.2$

Mean GRACE (death and MI) score

$124.3 \pm 44.0$

Median TIMI score (interquartile range)

$3.0(2.0)$ 


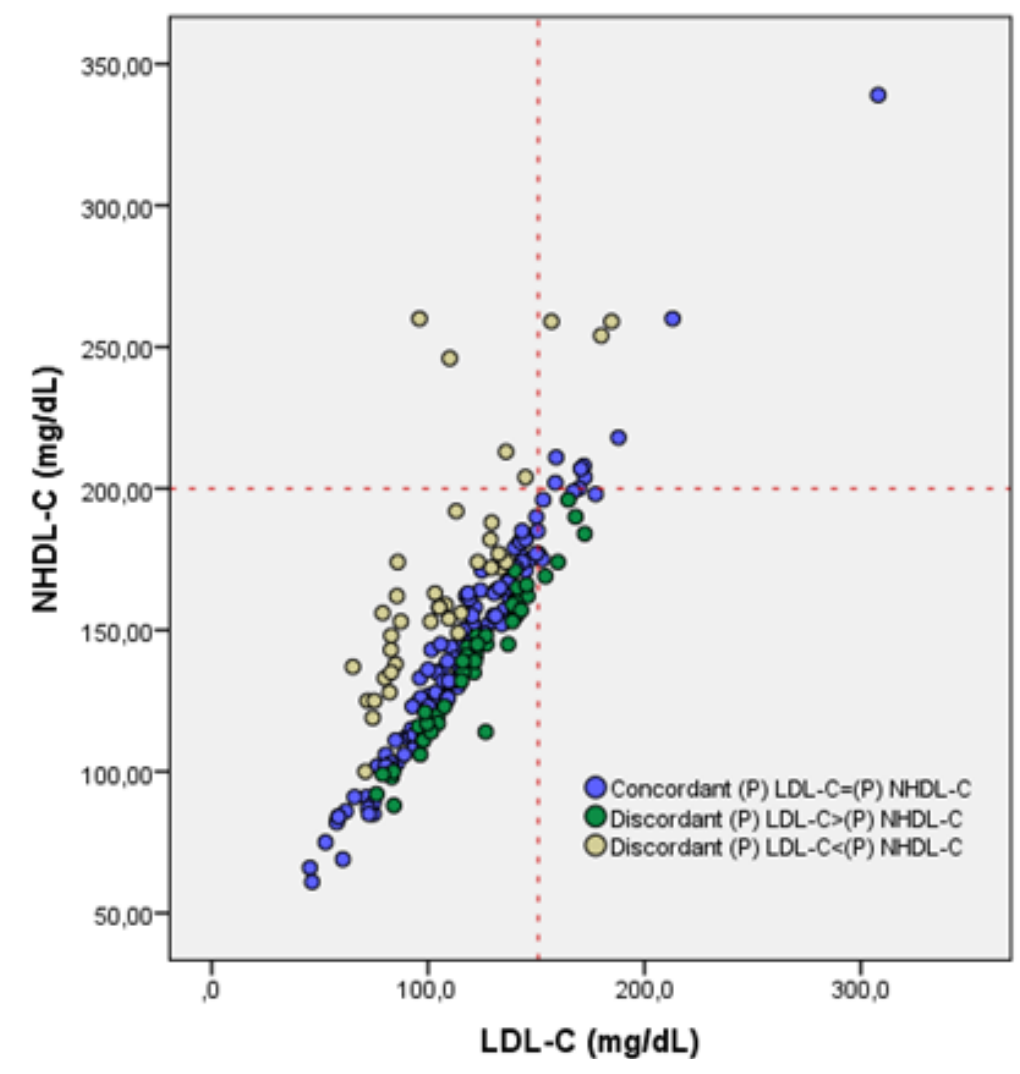

Figure 1 - Scatterplots and prevalence of discordance and concordance defined according to percentiles of LDL-C and NHDL-C.

It has been demonstrated that NHDL-C is a predictor of cardiovascular disease mortality in individuals free of cardiovascular disease. ${ }^{8-10}$ Also, it is associated with mortality in patients with coronary heart disease. ${ }^{11}$ However, the studies specifically evaluated its role on cardiovascular mortality after an index myocardial infarction were limited. In one study, it has been reported that NHDL-C $>130 \mathrm{mg} / \mathrm{dl}$ during follow-up after a myocardial infarction was associated with higher risk of long-term major cardiovascular events (MACE). ${ }^{3}$ Besides all-cause death, myocardial infarction, stroke and cardiovascular hospitalization were other components of MACE in that study.

LDL-C is the main target for lipid lowering therapy after myocardial infarction in daily clinical practice. ${ }^{1}$ In a recent study, LDL-C was found to be independently associated with increased cardiovascular disease mortality in individuals free of cardiovascular disease in 27-year follow-up period. ${ }^{12}$ It has also been demonstrated that LDL-C levels predict coronary heart disease mortality in men with preexisting cardiovascular disease..$^{13,14}$
However, studies evaluating LDL-C on prognosis after myocardial infarction have mixed results. In 2476 patients hospitalized for MI, small dense LDL was associated with low cardiovascular mortality. ${ }^{15}$ In a study by Reddy et al. the lowest LDL-C levels were associated with highest risk of in-hospital mortality after myocardial infarction. ${ }^{16}$

Sizeable proportion of patients have discordant LDL-C and NHDL-C (high LDL-C and low NHDL-C, and vice versa). Discordance rate changes from $11 \%$ to $29 \%$ in previous studies. ${ }^{4,17,18}$ It has been shown that discordance of LDL-C and NHDL-C either underestimates or overestimates coronary risk defined as non-fatal MI, percutaneous coronary intervention, coronary artery bypass grafting or coronary death. ${ }^{4}$ It was suggested that the LDL-C alone may give a false sense of security with one fifth of the subjects still having high cardiovascular risk, despite having normal LDL-C levels. ${ }^{19}$ Studies evaluating the impact of discordance of LDL-C and NHDL-C on coronary heart disease prognosis are limited and to the best of our knowledge, this is the first study to investigate the association between discordance of 
Table 2 - Characteristics of patients with concordant and discordant LDL-C and NHDL-C

\begin{tabular}{|c|c|c|c|c|}
\hline & $\begin{array}{c}\text { LDL-C }<\text { NHDL-C } \\
\text { n=38 (group 1) }\end{array}$ & $\begin{array}{c}\text { LDL-C=NHDL-C } \\
\text { n=112 (group 2) }\end{array}$ & $\begin{array}{c}\text { LDL-C }>\text { NHDL-C } \\
\text { n=48 (group } 3)\end{array}$ & p \\
\hline Age (years) * & $58.9 \pm 9.3$ & $63.5 \pm 11.1$ & $63.7 \pm 10.4$ & 0.054 \\
\hline Gender $(\% \text { male })^{\beta}$ & 73.7 & 70.5 & 81.3 & 0.369 \\
\hline Smoking $(\%)^{\beta}$ & 68.4 & 57.1 & 66.7 & 0.331 \\
\hline Hypertension $(\%)^{\beta}$ & 60.5 & 64.3 & 52.1 & 0.351 \\
\hline Diabetes $(\%)^{\beta}$ & 50.0 & 25.9 & 25.0 & $0.013^{a, b}$ \\
\hline Dyslipidemia $(\%)^{\beta}$ & 50.0 & 35.7 & 33.3 & 0.221 \\
\hline Body mass index $\left(\mathrm{kg} / \mathrm{m}^{2}\right)^{*}$ & $27.9 \pm 6.2$ & $26.2 \pm 4.5$ & $25.1 \pm 4.1$ & 0.376 \\
\hline Total cholesterol $(\mathrm{mg} / \mathrm{dl}){ }^{*}$ & $200.8 \pm 40.5$ & $182.8 \pm 44.7$ & $180.9 \pm 30.8$ & $0.044^{a, b}$ \\
\hline LDL-C $(\mathrm{mg} / \mathrm{dl})^{\delta}$ & $105.2(46.0)$ & $115.7(46.8)$ & $121.8(40.2)$ & 0.053 \\
\hline HDL-C $(\mathrm{mg} / \mathrm{dl})^{\delta}$ & $30.0(6.0)$ & $35.0(9.0)$ & $42.0(17.0)$ & $<0,001^{\mathrm{a}, \mathrm{b}, \mathrm{c}}$ \\
\hline Triglyceride * & $321.2 \pm 164.9$ & $140.1 \pm 43.8$ & $90.0 \pm 24.6$ & $<0.001^{\mathrm{a}, \mathrm{b}, \mathrm{c}}$ \\
\hline NHDL-C $(\mathrm{mg} / \mathrm{dl})^{\delta}$ & $158.5(37.0)$ & $147.0(53.5)$ & $141.0(42.5)$ & $0.002^{a, b}$ \\
\hline Mean GRACE (death) ${ }^{* * *}$ score ${ }^{*}$ & $114.5 \pm 29.5$ & $98.7 \pm 22.1$ & $112.0 \pm 30.3$ & $0.029^{a, b}$ \\
\hline Mean GRACE (death and MI) ${ }^{* *}$ score ${ }^{\delta}$ & $133.4 \pm 41.5$ & $104.3 \pm 31.1$ & $127.0 \pm 46.9$ & $0.008^{a, b}$ \\
\hline TIMI risk score ${ }^{\delta}$ & $3.0(2.0)$ & $3.0(2.0)$ & $3.0(2.0)$ & 0.925 \\
\hline In-hospital mortality (\%) & $1(2.6)$ & $2(1.8)$ & $1(2.1)$ & ---- \\
\hline Mortality at sixth month $(\%)^{\beta}$ & 5.7 & 5,7 & 4.4 & 0.176 \\
\hline Cardiovascular events at sixth month (\%) & 11.2 & 18.4 & 19.6 & 0.473 \\
\hline \multicolumn{5}{|c|}{$\begin{array}{l}\text { Data are expressed as mean } \pm \text { standard deviation or median(interquartile range) for continuous variables and number (percentage) for categorical variables, } \\
\text { *: One Way Anova, } \boldsymbol{\delta} \text { : Kruskal Wallis, } \beta \text { : Chi-square. LSD or Conover-Iman test was performed for the binary comparisons among the groups and the } \\
\text { p value was set at } 0.05 \text {. Significant differences were found between a: group } 1 \text { vs group } 2, \text { b: group } 1 \text { vs group 3, c: group } 2 \text { vs group 3. Statistically } \\
\text { significant p-values are in bold. }\end{array}$} \\
\hline
\end{tabular}

LDL-C and NHDL-C and widely used prognostic risk scores in MI patients.

Patients with AMI are heterogeneous in terms of clinical presentation, risk factors and prognosis. Identifying patients at higher risk for recurrent cardiovascular events and death after AMI is important to manage cardiovascular care. GRACE and TIMI risk scores are the most widely used risk scores for prediction of prognosis in AMI. In a study by Guler et al., ${ }^{20}$ lipoprotein(a) levels were higher in the group of patients with high GRACE score than in the group with intermediate or low GRACE score, and lipoprotein(a) was found to have an additional prognostic value over GRACE score in predicting oneyear adverse outcomes in NSTEMI patients. ${ }^{20}$ In the present study, we found that GRACE score is highest in the group with LDL-C $<$ NHDL-C compared to groups with LDL-C=NHDL-C and LDL-C $>$ NHDL-C. One of the reasons for low LDL-C and high NHDL-C is the 
amount of remnant cholesterol and lipoprotein(a) in plasma. Increased remnant cholesterol is associated with increased incident coronary heart disease and all cause mortality in general population. ${ }^{21,22}$ It is also associated with increased all-cause mortality in patients with ischemic heart disease. ${ }^{23}$ On the contrary, in a study with AMI patients, remnant cholesterol was found to be associated with low mortality. ${ }^{24}$ In our study, we didn't have data on patients' lipoprotein(a) levels and directly measured remnant cholesterol. However, TG was highest in the group with LDL-C $<$ NHDL-C and TG is marker of remnant cholesterol. ${ }^{25}$ Remnant cholesterol such as VLDL and IDL, as well as lipoprotein(a), is also atherogenic and associated with inflammation. ${ }^{26,27}$ Increased atherogenicity and increased inflammation may be related to high GRACE score in discordant group with increased remnant cholesterol. In a recent trial, lowering TG provides additional mortality benefit in patients with cardiovascular disease and using statin. ${ }^{28}$

\section{Study Limitations}

This study has several limitations. It is a retrospective study and there is the possibility of bias from unmeasured cofounders. There is no absolute definition and standard cut-off values for the discordance of LDL-C and NHDL-C. Other limitations include small sample size and short duration follow up. Further large-scale prospective studies will be required to validate our results.

\section{Conclusion}

GRACE score was higher in discordant group with LDL-C $<$ NHDL-C, but there is no difference regarding
TIMI scores between discordant and concordant groups. Risk stratification in AMI is mandatory to guide its management and patients with discordant LDL-C and NHDL-C may represent a group with poor prognosis. Individuals with AMI and discordance may be the target of the most aggressive pharmaceutical interventions.

\section{Author Contributions}

Conception and design of the research: Eren $M$, Kurmus O. Acquisition of data: Eren M, Aslan T, Akbuga $\mathrm{K}$, Tolunay H. Analysis and interpretation of the data: Eren M, Kurmus O, Aslan T, Akbuga K, Tolunay H. Statistical analysis: Eren M, Kurmus O. Writing of the manuscript: Eren M, Kurmus O. Critical revision of the manuscript for intellectual content: Kurmus O, Aslan T, Akbuga $\mathrm{K}$, Tolunay $\mathrm{H}$.

\section{Potential Conflict of Interest}

No potential conflict of interest relevant to this article was reported.

\section{Sources of Funding}

There were no external funding sources for this study.

\section{Study Association}

This study is not associated with any thesis or dissertation work.

\section{Ethics Approval and Consent to Participate}

This article does not contain any studies with human participants or animals performed by any of the authors.

\section{References}

1. Mach F, Baigent C, Catapona AL, Koskinas KC, Casula M, Badimon L, et al. 2019 ESC/EAS guidelines for the management of dyslipidaemias: Lipid modification to reduce cardiovascular risk. Eur Heart J. 2020; 41(1):111-8.

2. Sampson UK, Fazio S, Linton MF. Residual cardiovascular risk despite optimal LDL cholesterol reduction with statins: the evidence, etiology, and therapeutic challenges. Curr Atheroscler Rep. 2012;14(1):1-10.

3. Wongcharoen W, Sutthiwutthichai S, Gunaparn S, Phrommintikul A. Is non-HDL-cholesterol a better predictor of long-term outcome in patients after acute myocardial infarction compared to LDL-cholesterol?: a retrospective study. BMC Cardiovasc Disord. 2017;17(1):10.

4. Mora S, Buring JE, Ridker PM. Discordance of low-density lipoprotein (LDL) cholesterol with alternative LDL-related measures and future coronary events. Circulation 2014; 129(5):553-61.

5. Corcoran D, Grant P, Berry C. Risk stratification in non-ST elevation acute coronary syndromes: Risk scores, biomarkers and clinical judgment. J Cardiol Heart Vasc. 2015;8:131-7.

6. Antman EM, Cohen M, Bernink PJ, McCabe CH, Horacek T, Papuchis $\mathrm{G}$, et al. The TIMI risk score for unstable angina/non-ST elevation MI: A method for prognostication and therapeutic decision making. JAMA. 2000;284(7):835-42.

7. Friedewald WT, Levy RI, Fredeickson DS. Estimation of the concentration of low-density lipoprotein cholesterol in plasma, without use of the preparative ultracentrifuge. Clin Chem. 1972;18(6):499-502.

8. Ito T, Arima H, Fujiyoshi A, Miura K, Takashima N, Ohkubo T, et al Relationship between non-high-density lipoprotein cholesterol and the long-term mortality of cardiovascular diseases:NIPPON DATA 90. Int J Cardiol. 2016;220:262-7.

9. Harari G, Green MS, Magid A, Zelber-Sagi S. Usefulness of Non-HighDensity Lipoprotein Cholesterol as a Predictor of Cardiovascular Disease Mortality in Men in 22-Year Follow-Up. Am J Cardiol. 2017;119(8):1193-8. 
10. Cui Y, Blumenthal RS, Flaws JA, Whiteman MK, Langenberg P, Bachorik PS, et al. Non-high-density lipoprotein cholesterol level as a predictor of cardiovascular disease mortality. Arch Intern Med. 2001;161(11):1413-9.

11. Liao P, Zeng R, Zhao X, Guo L, Zhang M. Prognostic value of non-highdensity lipoprotein cholesterol for mortality in patients with coronary heart disease: A systematic review and meta-analysis. Int J Cardiol. 2017;227:950-5.

12. Abdullah SM, Defina LF, Leonard D, Barlow CE, Radford NB, Willis BL, et al. Long-Term Association of Low-Density Lipoprotein Cholesterol With Cardiovascular Mortality in Individuals at Low 10-Year Risk of Atherosclerotic Cardiovascular Disease. Circulation. 2018 ;138(21):2315-25

13. Pekkanen J, Linn S, Heiss G, Suchindran CM, Leon A, Rifkind BM, et al Ten-year mortality from cardiovascular disease in relation to cholestero level among men with and without preexisting cardiovascular disease. N Eng J Med. 1990;322(24):1700-7.

14. Van den Berg MJ, van der Graaf Y, de Borst GJ, Kappelle LJ, Nathoe HM, Visseren FLJ; SMART Study Group. Low-Density Lipoprotein Cholesterol, Non-High-Density Lipoprotein Cholesterol, Triglycerides, and Apolipoprotein B and Cardiovascular Risk in Patients With Manifest Arterial Disease. Am J Cardiol. 2016 ;118(6):804-10.

15. Pokharel Y, Tang Y, Bhardwaj B, Patel KK, Qintar M, O'Keefe JH Jr, et al. Association of low-density lipoprotein pattern with mortality after myocardial infarction: Insights from the TRIUMPH study. J Clin Lipidol. 2017;11(6):1458-70.

16. Reddy VS, Bui QT, Jacobs JR, Begelman SM, Miller DP, French WJ; Investigators of National Registry of Myocardial Infarction (NRMI) 4b-5. Relationship between serum low-density lipoprotein cholesterol and in-hospital mortality following acute myocardial infarction (the lipid paradox). Am J Cardiol. 2015;115(5):557-62.

17. Zhang Y, Wu NQ, Li S, Zhu CG, Guo YL, Qing P, et al. Non-HDL-C is a better predictor for the severity of coronary atherosclerosis compared with LDL-C. Heart Lung Circ 2016; 25:975-81.

18. Baruch L, Chiong VJ, Agarwal S, Gupta B. Discordance of Non-HDL and Directly Measured LDL Cholesterol: Which lipid measure is preferred when calculated LDL is inaccurate? Cholesterol. 2013;2013:502948.

19. Modi KD, Chandwani R, Ahmed I, Kumar KV. Discordance between lipid markers used for predicting cardiovascular risk in patients with type 2 diabetes. Diabetes Metab Syndr. 2016 ;10(1): 99-102.
20. Guler E, Gecmen C, Guler GB, Karaca O, Agus HZ, Gunes HM, et al. Adding lipoprotein(a) levels to the GRACE score to predict prognosis in patients with non-ST elevation acute coronary syndrome. Kardiol Pol. 2013;71(7):695-701.

21. Joshi PH, Khokhar AA, Massaro JM, Lirette ST, Griswold ME, Martin SS, et al. Lipoprotein Investigators Collaborative (LIC) Study Group. Remnant Lipoprotein Cholesterol and Incident Coronary Heart Disease: The Jackson Heart and Framingham Offspring Cohort Studies. J Am Heart Assoc. 2016;5(5):e002765.

22. Varbo A, Freiberg JJ, Nordestgaard BG. Extreme nonfasting remnant cholesterol vs extreme LDL cholesterol as contributors to cardiovascular disease and all-cause mortality in 90000 individuals from the general population. Clin Chem. 2015;61(3):533-43.

23. Jepsen AM, Langsted A, Varbo A, Bang LE, Kamstrup PR, Nordestgaard BG. Increased Remnant Cholesterol Explains Part of Residual Risk of All-Cause Mortality in 5414 Patients with Ischemic Heart Disease. Clin Chem. 2016;62(4):593-604.

24. Martin SS, Faridi KF, Joshi PH, Blaha MJ, Kulkarni KR, Khokhar AA, et al. ipoprotein Cholesterol and Mortality After Acute Myocardial Infarction: Further Evidence for a Hypercholesterolemia Paradox From the TRIUMPH Registry. Clin Cardiol. 2015;38(11):660-7.

25. Varbo A, Benn M, Tybjaerg-Hansen A, Jorgensen AB, Frikke-Schmidt R, Nordestgaard BG. Remnant cholesterol as a causal risk factor for ischemic heart disease. J Am Coll Cardiol. 2013;61(4):427-36.

26. Bernelot Moens SJ, Verweij SL, Schnitzler JG, Stiekema LCA, Bos M, Langsted A, et al. Remnant cholesterol elicits arterial wall inflammation and a multilevel cellular immune response in humans. Arterioscler Thtomb Vasc Biol. 2017;37(5):969-75.

27. Varbo A, Benn M, Tybjaerg-Hansen A, Nordestgaard BG. Elevated remnant cholesterol causes both low-grade inflammation and ischemic heart disease, whereas elevated low-density lipoprotein cholesterol causes ischemic heart disease without inflammation. Circulation. 2013;128(12):1298-309.

28. Bhatt DL, Steg PG, Miller M, Brinton EA, Jacobson TA, Ketchum $\mathrm{SB}$, et al.; REDUCE-IT Investigators. Cardiovascular Risk Reduction with Icosapent Ethyl for Hypertriglyceridemia. N Engl J Med. 2019;380(1):11-22. 